\title{
Talent development environment in a professional football club in Norway
}

\author{
Georg Flatgård ${ }^{1}$, Carsten Hvid Larsen ${ }^{2}$, \& Stig Arve Sæther ${ }^{*}$ \\ ${ }^{1}$ Norwegian University of Science and Technology, ${ }^{2}$ University of Southern Denmark
}

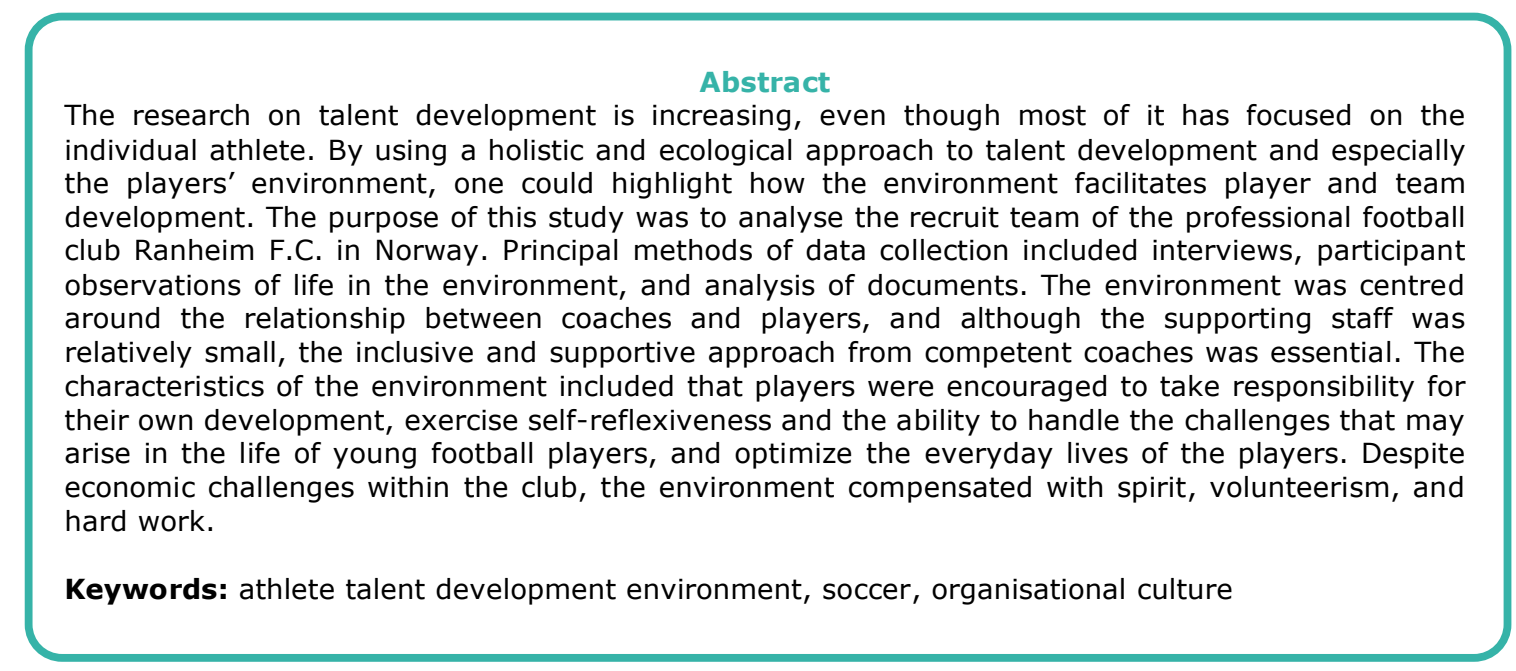

\section{Introduction}

Professional football is all about results and eventually winning trophies. Even so, the importance of developing local talented players is also an important feature for most clubs, even though some clubs are more successful on that matter. Earlier studies have found differences between selected and non-selected players in areas such as physiological skills (Huijgen, Elferink-Gemser, Lemmink, \& Visscher, 2014), dribbling and ball control (Figueiredo, Goncalves, Coelho e Silva, \& Malina, 2009), and psychological skills such as motivation (Forsman, Blomqvist, Davids, Liukkonen, \& Konttinen, 2016) and selfreferential cognitions (Höner \& Feichtinger, 2016). Even though many clubs select the most talented players at an early age, the high dropout rate (Güllich, 2014) indicates the challenges of identifying the future professional players early on. Despite the large amount of research on talent development that is centred around the earlier mentioned features of the players, there is also an increasing focus on the role of the environment and the cases that are successful in supporting young players' transition to the professional level (Côté, Baker, \& Abernethy, 2007; Stambulova, 2009; Stambulova \& Alfermann, 2009; Stambulova, Alfermann, Statler, \& Cote, 2009).

The athletic talent development environment (ATDE) was introduced by Henriksen, Stambulova, and Roessler (2010a) as a holistic ecological approach with a focus on the environment in which prospective elite athletes develop. This approach attributes considerable attention to the organizational context of the environment, and especially the role and function of the organizational culture. In talent development environment research, a strong and coherent organizational culture of the club or team has been linked to the environment's success (Henriksen et al., 2010a, 2010b; Larsen, Alfermann, Henriksen, \& Christensen, 2013). Studies from one professional Danish (Larsen et al., 2013) and one Norwegian (Aalberg \& Sæther, 2016) football club have found that the collaboration of the junior teams' development department was important for equipping young players with the resources needed, both on the pitch and in life in general. Their unified work methodology reduced the distance between U16 and U19 groups in the clubs because of these groups' close relationships. Knowledge of the next step is important for a smooth transition to elite-level football. Furthermore, the clubs highlighted the players' accountability of development as more important than the team's performance. Off the field, the club had tight relationships with a collaborative school, previously highlighted as crucial (Bourke, 2003; Christensen \& Sørensen, 2009). Paradoxically, there was a lack of relationship between the youth team and the senior team, as found in other football academies (Relvas, Littlewood, Nesti, Gilbourne, \& Richardson, 2010). The lack of such a relationship was also a reason for conducting a study on a similar environment to see if the same problem was present in other environments. The case chosen for the present study is a club which has a professional team which the last few years has been playing in the Norwegian premier league, and with few exceptions only includes players from the region of Trondelag (44 373,3 km², population 458.744). Even so, few of these players have made the transition from the recruit team to the 
professional team. The environment has, however, started a new approach trying to minimise the gap between the recruit team and the professional team. With the aim of increasing the number of players recruited from the recruit team to the professional team, this study focused on the male recruit team (27 players) and its staff ( 2 head coaches, one part-time physical coach and two physiotherapists working on a voluntary basis) in the professional football club Ranheim Football Club. The aims of this study were to present a holistic ecological description of Ranheim Football Club's recruit team and to describe the organizational culture of the club's recruit team.

\section{Methods}

The study was organized as an explorative integrative and qualitative oriented case study in a potentially successful Norwegian football environment (Maaløe, 2004). A case study is an empirical method that examines a contemporary phenomenon in its actual context, which is the method's major strength according to Flyvbjerg (2006), since this causes the researcher's assumptions to be tested based on actual events as they occur. A case study could therefore be seen as a good way to create fertile knowledge of a complex phenomenon that occurs in its natural context (Yin, 2009). With the use of an exploratory and integrative approach to case studies, these should, according to Maaløe, be carried out as a 'cyclical approach' with continuous dialogue between predefined theories, generated data, the researchers' interpretations and feedback from informants' (Maaløe, 2004, p. 3). Because our objective was to describe a specific environment and understand its potential success, we expected that the case study would be an appropriate methodological choice for this study. More specific it is a maximum variation case (Flyvbjerg 2006) because the selected club was very different from comparative professional clubs in terms of budget and its newly re-organised structure related to the recruit team and their approach on player development.

\section{Case selection and participants}

The study adopts the holistic ecological approach established by Henriksen et al. (2010a), introduced as a case study of the development environment of the recruit team of Ranheim Football Club. The recruit team consists of the players who are closest to playing for the professional team. The club also has a U19 team, but this U19 team is open to all players who wish to play for Ranheim Football Club. The slogan Ranheim uses related to its talent development is "We realise dreams", and symbolises that the club gives young talented football players the opportunity to realise them. The professional team of Ranheim has been a professional team since it was promoted to the Norwegian level 2 (obos-league) in 2010. In 2017 it qualified for the Norwegian level 1 league and has since 2018 played in the top level league (Eliteserien).

Furthermore, Ranheim is considered both by itself and also by most people who follow football as the little brother of football in Trondheim, where the big brother is the well-known Rosenborg Football Club. As a consequence of Rosenborg's new policy on its junior team (keeping fewer players in the squad), Ranheim has recruited a lot of the players who have left Rosenborg. At the time of the study Ranheim had recruited 17 players and three coaches (though not at the same time). Despite the positive side of harder competition and a higher level of the training sessions, this could lead to internal rivalry.

This environment has somewhat recently been established in its current form, and this is therefore a feature of the environment and should be used as a basis for understanding the environment. A special feature of Ranheim Football Club is the fact that it declined to be a part of the academy classification within Norwegian toplevel football. Only two out of 32 teams declined the invitation.

\section{Research methods and instruments}

Data was collected from three main sources (qualitative interviews, participant observation, and analysis of documents) and from multiple perspectives (peers, related teams, coaches, and players in the club), and was conducted by the first author.

Interview

Firstly, semi-structured interview guides (Kvale \& Brinkman, 2009) were created on the basis of the predisposed categories from the ATDE and ESF models (Henriksen, 2010b). Two in-depth interviews were conducted, one with a recruit player and one with a head coach, lasting 80 and 120 minutes. These were chosen because of their role in the environment where the selected coach had been the longest time in the club and the selected player, was one of the players closest to making the transition to the professional team.

Participant observation

Secondly, participant observations were used as a method (Tanggaard, 2006), with a mix of passive observation and moderate observation (Spradley, 1980). The observations took place at the clubhouse, the training grounds, and at matches. Altogether the observations covered about 40 hours (two separate weeks of observations) across two months and included informal conversations with parents, coaches, administrators, elite players, volunteers, and teachers.

Analysis of documents

Thirdly, archives and documents were used as a substantial category of data in the case study (Ramian, 2007). The documents included the club's homepage; official papers describing the vision, rules, and structure of the club; and statements about its vision, rules, goals, strategies, and values.

Data treatment and analysis

All interviews were audio recorded. Interviews and observation notes were transcribed verbatim. Raw material was coded using a deductive categorization of the data (Kvale \& Brinkmann, 2009). The deductive coding was based on a node tree built from the ATDE and the ESF models and primarily involved high-order themes.

\section{Findings}

Description of the Ranheim Football Club as a talent development environment

Microenvironment

The study focused on the recruit team (27 players) and its staff in the professional football club Ranheim FC (see Figure 1).

The staff. The coaches related to the group are mainly two head coaches, but the group also includes one physical coach and two physiotherapists working on a voluntary basis. The coaches seemed to have a close relationship with the players.

Even though the coaches want to provide the players with a good environment for training and development, they also challenge the players, expecting them to take ownership of their own development, so the players have to own the process, but facilitated by the coaches; this was a strategic approach from the coaches. One of the coaches highlighted this, saying, 'Handle adversity, handle prosperity. . . Holistic player development . . . including the 


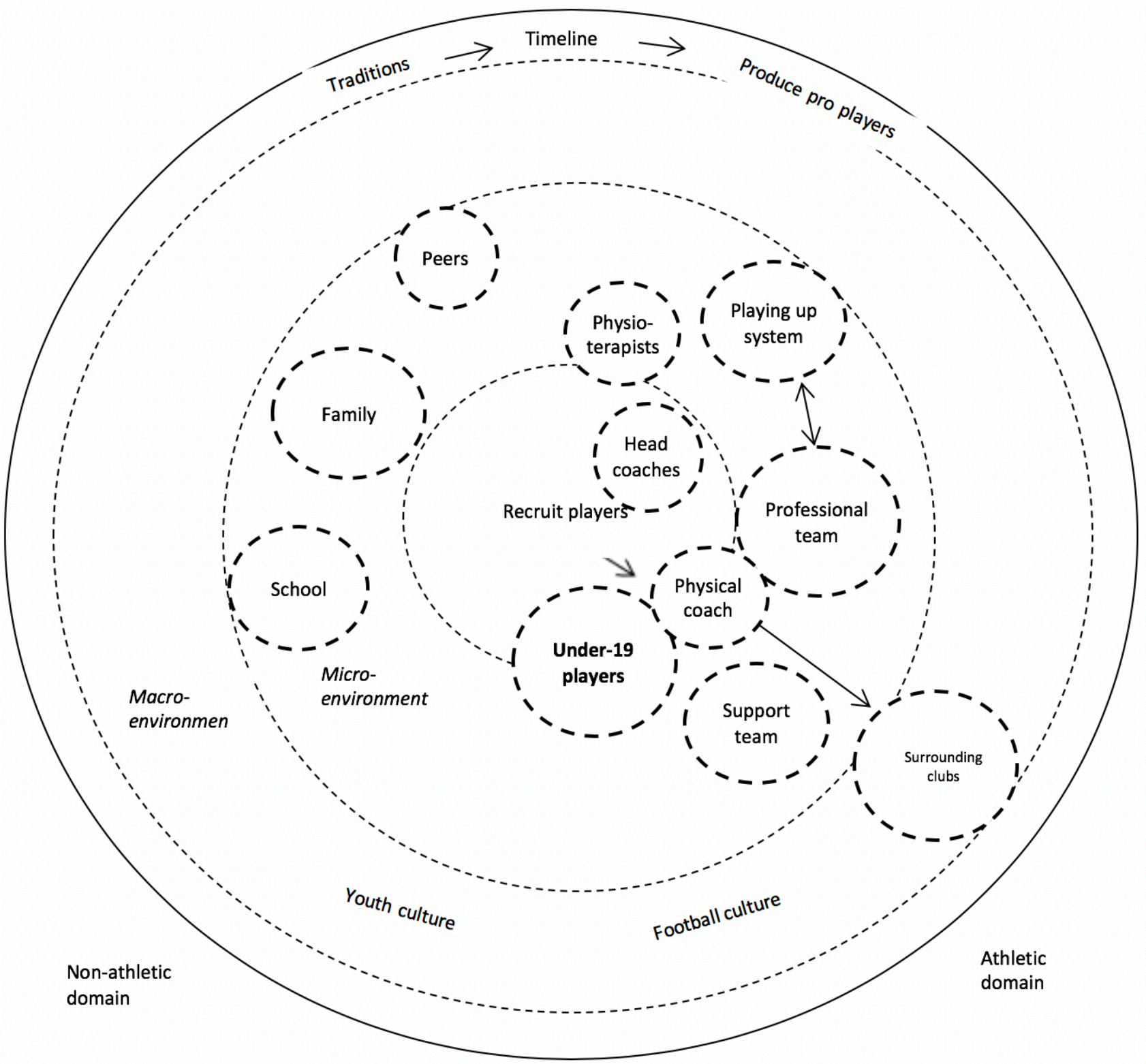

Figure 1: ATDE empirical model of Ranheim Football Club

social aspect. We want to develop the whole player.' (Coach $1)$.

Ranheim naturally has fewer resources compared to other professional clubs it competes with (i.e., Rosenborg FC) (Aalberg \& Sæther, 2016). The advantage of having two coaches would be the likelihood of communicating the same messages by communicating the same message in different ways. However, these two coaches also had quite a few other roles included in their positions: player developer, head of analysis, and coach mentor. This gave them the opportunity to have a holistic approach to player development, and potentially as a consequence they were not occupied with details and trying to develop certain types of players, instead highlighting the tactical component of the game. This approach could be seen as quite bold, but the fact that both coaches have a master's degree in sport science could give the coaches safety on the basis for their approach.

Under 19. Naturally, the under-19 team is the group closest to the recruitment team. The fact that the under-19 team (approximately 35 players) is open to every player wanting to play could be understood as something unusual in a professional club, but the coaches argued for the importance of the current arrangement as follows: 'If we haven't got the ordinary team, then we had to be the ordinary team. We can't be a club without a team for the best players among the junior players.' (Coach 1 ). The term used on the teams in the club have been under debate and have created some confusion or at least some misunderstandings.

Playing at a higher age: a system? Letting players play with older players (from this point we use the term "playing up") is a vital part of the club's player development, even though there is not a systematic approach in the club. Independent of the senior team coaches and their needs (there is no official list of players), some players have been training and have played matches with the professional senior team (especially in pre-season). However, the club has two different methods, which they call random playing up and accrued playing up. Naturally enough, random playing up is characterised by an apparent need in the senior team. As observed, playing up was not given to the player with the 
highest potential, but to an overall assessment of the players, consequently living up to a holistic approach to player development. The overall priority seems to be the overall load, including school, match load, and training load and injuries, but also including the social aspect (players felt socially safe), and having a social toughness to be able to handle being a part of a senior team training session. There also were examples of periodic/accrued playing up with the senior team (weeks, months, or regularly), where the senior coaches were responsible for follow-up and feedback. Even if these playing up opportunities most often were expected to get the players more motivated and inspired, some of the players got frustrated, because of the uncertainty: 'If I get the opportunity to play up regularly with the senior team it is okay to stay in the club, but I should get some promises of playing up. I don't get much information about that.' (Player 5, field talk). It seemed positive, however, that there was not only a small group of players playing up, but that each player had an individual development plan (together with the coaches), which was hung over each of the players' seats in the locker room. Many of the players had the main goal of "training with the senior team".

Senior team. The relationship with the senior team seemed quite close, and this was highlighted as important by the coaches for both the recruit team and the senior team. Both, however, also highlighted that there were different levels of performance, but they make sure that the players are fully invested in things unrelated to performance level, such as will and effort. The senior team most often trained before the recruit team on the same pitch or a connected pitch, and the players from both teams were often observed greeting each other. On one occasion the coach and the captain of the senior team were seen observing the warmup of the recruit team. This was also observed by one of the coaches on the recruit team: 'Naturally the recruit players see that. It would not happen in Rosenborg, I think. Being so close. It becomes another closeness, also to the level of reference.' (Coach 2, field talk). Ranheim seemed to have a focus on knitting these teams together, using the senior team as a reference. This included video from the senior team matches, both for the team and also for individual players, as highlighted by one of the players: 'I would actually compare our recruit team with their senior team. At least compared to unity and togetherness. The senior team is a closeness; the senior team is a group of buddies from another world.' (Player 1). And even though the playing up was quite sporadic, it seemed realistic both in training and matches (10-12 players in pre-season). The same player said the following in terms of taking part in a training session on the senior team: ' $I$ think it is a good thing that the senior team greets the recruit players. . . Especially the youngest players on the senior team take care of us and we thereby feel a part of their group. It feels like they are your buddies even though you haven ' $t$ known them that long.' (Player 1).

Microenvironment (non-sport domain)

There were three relations seen as important in terms of peers, family, and school.

Peers. Being both a school pupil and a football player at a high junior level is a challenge, as many studies have confirmed, especially because of the lack of time for friends, which one of the coaches stated was a common reason for players to quit. This issue was furthermore observed by the first author, since he noticed that one of the players (described as potentially one of the best in the group) was absent in the recruit team for a short period. The coaches had therefore let the player train less in a period to see if the lack of motivation changed as a consequence of fewer training hours.
Family. Since the players were encouraged to take accountability for their own development, the coaches wanted to relate as little as possible with the parents. The communication with the parents was mainly managed on Facebook, highlighting what was going on with the team. Even though the coaches wanted as little as possible to do with the parents, the parents come in handy when the team needs help with both voluntary work and support for the team. Many of the players, however, highlighted the importance of the parents in supporting them, driving them to training, making food, being a supporter on matches, or being helpful when the players were doing self-organized training sessions.

School. Ranheim has no formal collaboration with an upper secondary school in the area, which means that the players are spread over four different schools, where most players attended a sport-specific school program. Both the players and the coaches highlighted the challenge of the total physical load, even though the football sessions on school was coordinated. The coaches and the teachers at the schools seemed well coordinated, and they acknowledged the players' situation, as one of the players stated: 'The school coach and the club coach have a close relationship when it comes to training load, and both parts are good at adjusting and regulating.' (Player 3 , field talk).

The collaboration with the physical education teachers, however, was another matter, where there often was a conflict when players asked to drop out of physical hard training sessions when they were playing games in the evening or at other locations.

The dilemma of optimizing the everyday training situation started a discussion related to the need for a school collaboration, while on the other hand not giving the players too much help in the process. The coach sees this challenge in another way and points to the fact that such a collaboration would mean that the players lack an excuse for not performing or developing as expected, since everyday life gets easier. Even so, there are obvious positive effects of such a collaboration, like a higher degree of flexibility in terms of training sessions, playing up, and assessment of the total load of physical activity.

Macroenvironment

Local clubs. Even though Ranheim has no formal collaboration with local clubs, it still invests a lot of time in collaborating with different local clubs. Despite the vision to fulfil the dreams of young talented players from the region, the club admitted that the road from the recruit team to the senior team was too long, and often included a loan to a local club. According to one of the coaches, the players were the most important element, not the club. But even if the coaches wanted to give the players challenges, the different players' development processes were different, and it was difficult to predict the players' future skills. One of the short goals of development in the club was to give players opportunities to develop, even if it meant playing for another club. 'If I could send a first-year senior or a lastyear junior to a second or third division club and they actually get playing time, it would be seen as a success for us.' (Coach 1 ).

Even though the club had a clear policy of recruiting local talented players from the region, the road seemed long for the players, which naturally could be frustrating, as one of the players stated: 'I think that a player we had who went to $(X X)$ may have done just as well as a player the senior team got from the junior team of Rosenborg. I believe Ranheim simply has to believe in the players they have produced. . . That could be a reason as to why there haven't been coming up that many players because of the ones picked up from Rosenborg.' (Player 1). 
Environment success factors (ESF)

The empirical version of the ESF model (see figure 2) summarizes the factors influencing the success of Ranheim Football Club as an ATDE.

Preconditions. The club has the lowest budget in the Eliteserien of approximately 2 million euro (17 million Norwegian kroner). To put this in perspective, Rosenborg's budget is ten times higher, and Vallerenga uses the same amount as Ranheim's total budget on support staff. In 2016 the club got a bankruptcy petition and was forced to sell a shareholding to be able to pay the treasurer. The club also had to save 2 million kroner in the last two quarters in 2016 on a budget of 10,5 million kroner, which had repercussions, mostly for the staff. As a consequence, in 2017 the senior team had two coaches and the recruit team had one head coach in a $50 \%$ position and an assistant coach in a $10 \%$ position. As an example of the club's financial situation, the recruit coach had to decline the opportunity to take a coach course provided by the Norwegian football federation. Another example of the economy in the club is the reuse of clothes: the recruit team players inherit the senior players' clothes-both team jerseys and training clothes-from the last season. Even if the situation was described as somewhat negative, this was not the impression the first author got when he attended the inherit process at a team meeting.

Facilities. The club has appropriate facilities, the recruit team has its own locker room that it has inherited from the senior team (leaving names from the senior team players on the benches). The fact that the team has its own locker room means that it can decorate it as it wishes, and it makes it easier for the players to meet up early for training and stay behind after a training session, which is something the players appreciate. The club also has built a new section of the stadium, including a training centre which a sponsor of the club has built. This makes strength sessions easier to do, and even more so for the players who have an injury and train alternatively. One of the main challenges is the number of pitches the club has access to: two full-size pitches and one seven-a-side pitch. As a consequence, the recruit team has to conduct two of its weekly training sessions at a pitch a few kilometres away. This is a pitch owned by the local football federation, so the team can train for free, but there are no locker room facilities at the pitch. The daily routine. The training and match schedule is different for the individual player, and it is mostly based on overall physical load. The coaches have tried to have an overall picture of the players, and since the

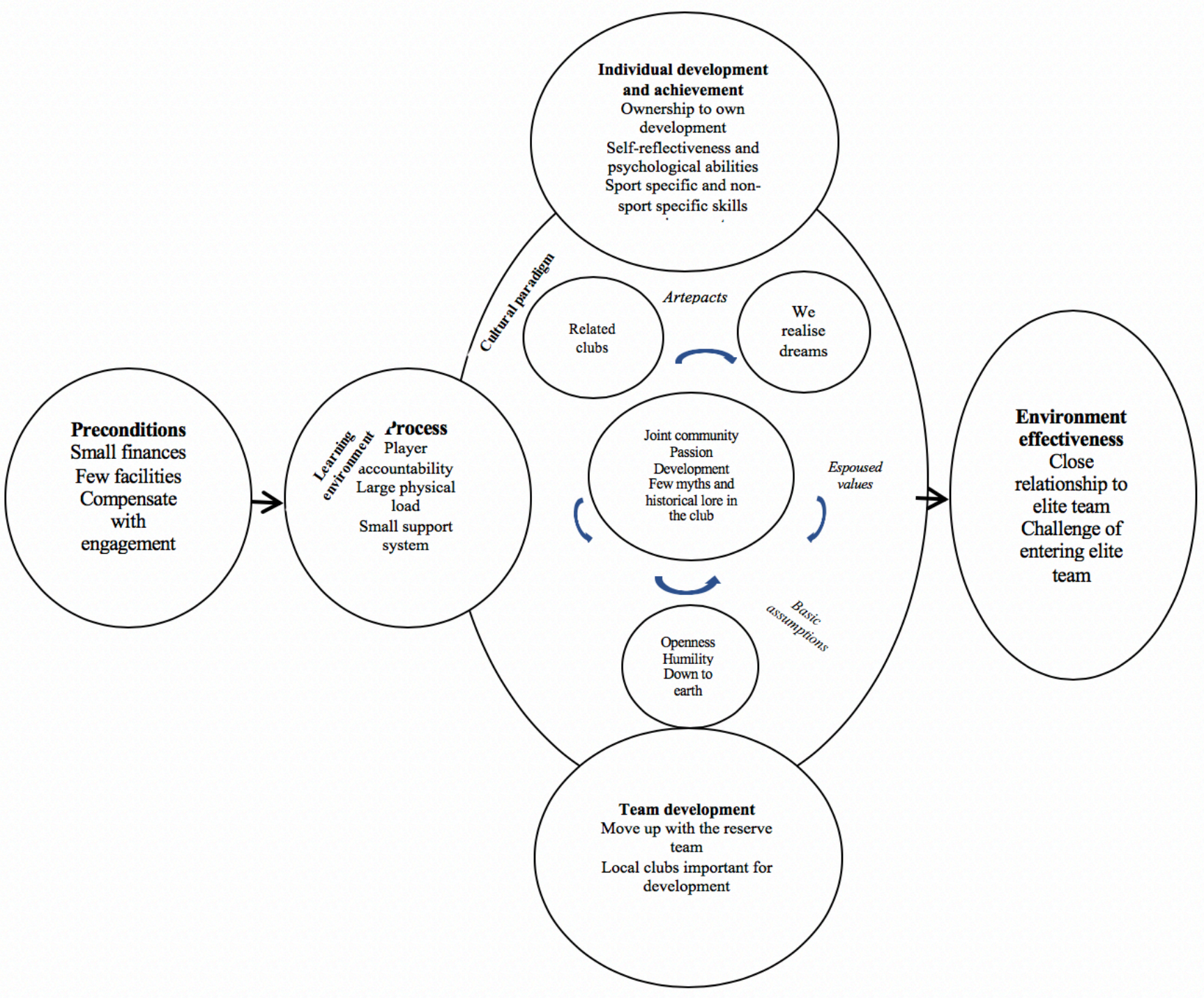

Figure 2: Empirical model of Ranheim Football Club 
physiotherapists are only volunteers and do not regularly attend all training sessions, they conduct a screening of basic skills, giving an indicator of which skills the players need to develop (muscular strength). Even so, in my observations the lack of a physiotherapist attending sessions was a problem, which was observed in the period and something the players wanted, even though this was highlighted as better than last year's season. As a compensation, most likely, the coaches use an RPE scale (Rate of Perceived Exertion) form, to track the players' overall physical load, ranging from 6 (no effort) to 20 (maximal effort); this form is completed every day for all types of activities. Lack of filling in this form on a daily basis was furthermore related to the players' will and ability to own their own development. As a consequence of the lack of collaboration with an upper secondary school, this form is seen as essential to be able to keep track of the players' overall physical activity. The importance of regulating the number of matches is furthermore essential to keep track of the load.

Cultural paradigm

Artefacts. The Ranheim slogan, "We realise dreams", symbolises that the club gives young talented football players the opportunity to realise their dreams. In the first game of the 2018 season, the club's first season in the Norwegian premier league, eight of the players were from the region. None of these players or the ones on the bench, however, had been a part of the club's youth department. The club was aware of the issue, and one of the coaches admitted that the players were in a difficult situation. The club tried to ship off the players to local clubs to give them experience, which was the dream scenario. The coaches tried to communicate this to the players, and one of the coaches said this on the matter: 'Because the fact of recruiting a player directly from the recruit team to the senior team seems so unrealistic because of the difference in level of performance in the elite series or the OBOS league (the name of the sponsor of the league) . . . there is one player each third year, and that statistic applies for most top-level clubs. ... We consider communicating that to the recruit players as important.' (Coach 1 ).

Espoused values. It seemed like Ranheim has quite well described what it wants to be as a club. It furthermore has stated three espoused values it works for: community, passion, and development. During the first author's observations, field talks, and interviews, he was given the impression that athletes, coaches, and managers in the environment really lived by these values. These values shone true in internal communication and also by people outside the club. Most acted humbly and appreciated the joint community they were a part of. The coaches and players from the senior team and the recruit team seemed to want to help each other, and the coaches used the term "family" frequently. As an example, when recruited players got to train with the senior team, the coaches made sure that the different exercises were done with a mix of age, so that the youngest players did not feel exposed but included. This was also seen in the locker room culture where laughter and a good atmosphere seemed to be present, which also was described by one of the players: 'I think we have the best wardrobe culture in Trondheim. All are buddies and talk to each other. There are a few cultural differences in the wardrobe; all the players are from Trondheim and that indicates that there is a small distance between us.' (Player 1).

The value of passion was a vital characteristic in the club, and it involved aspects that have no monetary cost: being proud of hard work and doing voluntary work. The value of development, however, was the most important characteristic in the club. The club seemed to have an eye for the potential for development in all parts of the club. All these three values were, however, in their written documents quite imprecise and general, which gives the club the opportunity to embrace many different visions and philosophies, even though these values seemed present in the environment. Stability seemed also to be important for the club, which indicated harmony and safety for both players and staff, leaving the opportunity for a long-lasting implementation of a philosophy and a culture which was supportive and coherent.

There seemed to be few myths and historical lore in the club. Compared to Rosenborg FC, the club has neither a successful story nor a legendary stadium, and the club could be described as having few cultural characteristics. Instead of referring to tradition, the club referred to routines. The clearest symbol was most likely the colour blue, so the club could be described as in the making, and it saw the importance of building an identity. The club's senior team tried to make a symbol of the club, and the new sponsor of the club had the symbol of a unicorn; this has been used to symbolise that Ranheim is something unique and that it is on an adventure.

Basic assumptions. Ranheim seemed to be a club based on approachability and a humble approach, both between the players and staff and external people coming into the environment (family feeling). The first author, even if he for a short period has been a part of the club as a player for the professional team, felt welcome and included in every aspect and all discussions, and this is something that was highlighted by one of the coaches: 'The door is open, it is down to earth, no one puts themselves over others. The material manager and the senior head coach are down to earth. I think that is quite nice compared to other clubs.' (Coach 1).

Another basic assumption was the fact that the sport plan for the club was detailed in describing the club's philosophy and the way the club wants to play football. These documents state that the playing system, principles, and roles are to be used all the way from the under-7 team to the senior team. Even if such a document has the potential not to be implemented but to only stay on the office table, since the recruit coaches are coach supervisors for other teams in the club, it made it easier to hold on to the philosophy. Naturally the playing pattern became more obvious as the players got older, and the similarities between the recruit team and senior team seemed especially present, potentially because of the fact that they shared both time and space and played together in matches.

Individual development and performance. In Ranheim there was a focus on identity, an open and honest approach, in which the individual player was given the opportunity to take responsibility for his own development. Essential in this approach was the coaches' responsibility to provide the players with the opportunity to become selfreflexive and able to make reasonable choices using individual development plans. This plan was based on discussion between the player and the coaches and highlighted goals, strengths, and adjustments which could help the player towards his goals.

The clubs wanted to educate the players related to their ability to think, tactical skills, and development of a specific understanding of their playing position in the team. As an example, the clubs would argue that all players should be playing for a longer period, because it was such an essential role to experience for the player, and furthermore it gives the coaches the opportunity to follow the players' development and see their standing as players.

Holistic development was essential and meant that the players, alongside their football-specific development, 
additionally should exploit their ability to use resources and tools necessary to succeed in life in general. The environment seemed to equate the importance of development as a player and development as a human, highlighting the mental skills which could come in handy both in a professional football career or in life in general. Team goals and development. All training sessions had a tactical outgrowth, which meant that all passing drills, possession drills, and game play drills were in focus. This also meant that the coaches used every game play drill as an opportunity to discuss and guide the players, often making the player come up with solutions. The training sessions included few technical components, which was a conscious action from the coaches, and the argument from the coaches was not to overload the players with tactical components which included obvious physical and technical components. This was a good prerequisite to developing holistic players.

Even if the club seemed to have a firm focus on the player's individual development, there were still some potential dilemmas and the balance between the individual and the team goals and development. The first author got to be a part of a team meeting discussing the team goals both for Ranheim 2 and the elite junior team. The focus was largely based on results, with little focus on development, while there was some focus on playing style. Some of the players also stated that they did not see the value of these team goals, and would rather have more goals on playing style. Since Ranheim 2 often consists of players from the professional team, the recruit team players get less playing time, which affects their opportunity to develop as footballers.

\section{Discussion}

The purpose of the study was to present a holistic ecological description of Ranheim Football Club's recruit team, and to describe the organizational culture of that team.

Although each successful ATDE is unique, the present study, as compared with previous studies, showed that the environment shares a number of factors contributing to its success. First, despite challenging economic preconditions, the club compensates with profound spirit, volunteering, and hard work. The environment was centred around the relationship between coaches and players, and although the supporting staff is relatively small, the staff's inclusive and supportive approach was essential. The close coachathlete relationship was furthermore seen as an important counterbalance for the lack of economic clout. Earlier studies have pointed out that this relationship has been shown to be important, giving the players good preconditions to be able to become professional players (Martindale, Collins, \& Daubney, 2005; Martindale, Collins, \& Abraham, 2007).

Secondly, the players were encouraged to take responsibility for their own development, highlighting selfreflexiveness and the ability to handle the challenges that may arise in the life of young football players, and optimizing their everyday lives. This was a strategic approach from the coaches, which also is supported by research, since mental skills (i.e., self-regulation, ability to work hard, and the ability to exploit social skills) are important for the players' development (Larsen, Alfermann, \& Christensen, 2012).

Third, a joint understanding of the way the club plays football was especially essential since the transition between junior and senior has been found to be challenging (Stambulova, 2009). Furthermore, studies have shown that coherence in communication and support could be decisive in successful talent development (Martindale et al., 2005, 2007).

Fourth, the recruit team and the professional team seemed to have a close relationship. Since earlier studies on environments in professional football clubs have found a lack of relationships between junior/recruit teams and senior teams (Larsen et al., 2013, Aalberg \& Sæther, 2016), Ranheim seems to have a higher focus on knitting these teams together, using the senior team as a reference. The club had a clear policy of letting the players play with older players, an approach which was done systematically.

While professional football is all about short-term results and eventually winning trophies, player development is on the contrary a long-term process. And even though scouting still is an important part of most professional club's development programs, the dropout rate is high (Güllich, 2014). So despite the fact that earlier research have found differences between selected and nonselected players on skills such as physiological skills (Huijgen, Elferink-Gemser, Lemmink, \& Visscher, 2014), dribbling and ball control (Figueiredo, Goncalves, Coelho e Silva, \& Malina, 2009), and psychological skills such as motivation (Forsman, Blomqvist, Davids, Liukkonen, \& Konttinen, 2016) and self-referential cognitions (Höner \& Feichtinger, 2016), they are less successful in predicting which players are going to reach professional football. Since Bloom (1985) introduced his model of talent development, there has been introduced a range of models trying to explain talent development processes (Gulbin, Croser, Morley, \& Weissensteiner, 2013). Henriksen (2010) was, however, one of the few who took a holistic ecological approach to talent development, introducing the ATDE working model. This study has with the use of this model described how the talent development environment of the recruit team of Ranheim could be seen as potentially successful in the years to come. The contribution of the present study gives an insight into how a newly reorganized talent development environment could be potentially successful based on their talent development approach highlighting the importance of holding the players as their core person and the environment as a catalyst to development by hard work, responsibility and a close player-coach relationship.

\section{Barriers for success}

The environment had some pivotal problems related to playing opportunities and the players' individual and team goals. Even though the club has a system for playing up, there were difficulties in terms of few playing opportunities for the recruit players on the professional team, making the transition from junior to senior difficult. Furthermore, there were issues related to the players' individual and team goals; there were examples where the team goals trumped the players' individual goals. Despite the club's distinct playing style and development model, the focus on performance is difficult to get away from even in an environment with the main focus of producing professional players.

Since the environment of the club was only recently established in its current form, the future will tell if it can manage to fulfil its slogan: "We realise dreams".

\section{Conclusion}

By using a holistic and ecological approach in the case of the professional football club Ranheim F.C. in Norway, we studied the club's recruit team and how this environment facilitated the players. The environment was centred around the relationship between coaches and players, and although the supporting staff is relatively small, the staff's 
inclusive and supportive approach was essential. The players were encouraged to take responsibility for their own development, highlighting self-reflexiveness and the ability to handle the challenges that may arise in the life of young football players, optimizing everyday lives for the players.
Despite challenging economic preconditions, the club compensates with profound spirit, volunteering, and hard work. Somewhat different from other professional football clubs, the recruit team and the professional first team seem to have a strong relationship.

\section{References}

Aalberg, R. R., \& Sæther, S. A. (2016). The talent development environment in a Norwegian top-level football club. Sport Science Review, 3-4, 159-182.

Bloom, B.S. (1985). Developing talent in young people. New York: Ballentine Books.

Bourke, A. (2003). The dream of being a professional soccer player: Insights on career development options of young Irish players. European Physical Education Review, 5, 121-131. Physical Education Review, 5, 121
doi:10.1177/0193732503255478

Christensen, M. K., \& Sørensen, J. K. (2009). Sport or school? Dreams and dilemmas for talented young Danish football players. European Physical Education Review, 15, 115-133. doi:10.1177/1356336X09105214

Côté, J., Baker, J., \& Abernethy, B. (2007). Practise and play in development of sport expertise. In G. Tenenbaum \& R. C. Eklund (Eds.), Handbook of sport psychology, (pp. 184-204). Hoboken, NJ: John Wiley \& Son.

Figueiredo, A. J., Goncalves, C. E., Coelho e Silva, M. J., \& Malina, R. M. (2009). Characteristics of youth soccer players who drop out, persist or move up. Journal of Sports Sciences, 27(9), 883-91.

Flyvbjerg, B. (2006). Five misunderstandings about case-study research Qualitative Inquiry, 12, 219-245.

Forsman, H., Blomqvist, M., Davids, K., Liukkonen, J., \& Konttinen, N. (2016). Identifying technical, physiological, tactical and psychological characteristics that contribute to career progression in soccer. International Journal of Sports Science \& Coaching, 11(4), 505-513. doi:10.1177/1747954116655051

Gulbin, J.P., Croser, M.J., Morley, E.J., \& Weissensteiner, J.R. (2013). An integrated framework for the optimisation of sport and athlete development: a practitioners approach. Journal of sports Sciences, 31, $12,1319-1331$

Güllich, A. (2014). Selection, de-selection and progression in German football talent promotion. European Journal of Sport Science, 14(6), 530-537. doi:10.1080/17461391.2013.858371

Henriksen, K., Stambulova, N., \& Roessler, K. K. (2010a). Holistic approach to athletic talent development environments: A successful sailing milieu. Psychology of Sport and Exercise, 11, 212-222.

Henriksen, K. (2010b). The ecology of talent development in sport: A multiple case study of

successful athletic talent development environments in Scandinavia. PhD dissertation, University of Southern Denmark.

Höner, O., \& Feichtinger, P. (2016). Psychological talent predictors in early adolescence and their empirical relationship with current and future performance in soccer. Psychology of Sport and Exercise, 25, 17-26.

Huijgen, B. C. H., Elferink-Gemser, M. T., Lemmink, K. A. P. M., \& Visscher, C. (2014). Multidimensional performance characteristics in selected and deselected talented soccer players. European Journal of Sport Sciences, 14(1), 2-10.

Kvale, S., \& Brinkmann, S. (2009). Interviews: Learning the craft of qualitative research interviewing. (2nd ed.). Thousand Oaks, CA: Sage Publications.

Larsen, C. H., Alfermann, D., \& Christensen, M. K. (2012). Psychosocial skills in a youth soccer academy: A holistic ecological perspective. Sport Science Review, 21(3-4), 51-74. doi:10.2478/v10237-012-0010-x

Larsen, C. H., Alfermann, D., Henriksen, K., \& Christensen, M. K. (2013). Successful talent development in soccer: The characteristics of the environment. Sport, Exercise, and Performance Psychology, 2(3), 190-206. doi:10.1037/a0031958

Maaløe, E. (2004). In case of case research. Aarhus: Department of Organization and Management, Aarhus University.

Martindale, R. J. J., Collins, D., \& Abraham, A. (2007). Effective talent development: The

elite coach perspective in UK sport. Journal of Applied Sport Psychology, 19, 187-206. doi:10.1080/10413200701188944

Martindale, R. J. J., Collins, D., \& Daubney, J. (2005). Talent development: A guide for practice and research within sport. Quest, 57, 353-375. doi:10.1080/00336297.2005.10491862

Ramian, K. (2007). Casestudiet i praksis [The case study in practice]. Aarhus: Academica.

Relvas, H., Littlewood, M., Nesti, M., Gilbourne, D., \& Richardson, D. (2010). Organizational structures and working practices in elite European professional football clubs: Understanding the relationships between youth and professional domains. European Sport Management Quarterly, 10, 165-187. doi:10.1080/16184740903559891

Spradley, J. P. (1980). Participant observation. Fort Worth, TX: Harcourt Brace College Publishers.

Stambulova, N. (2009). Talent development in sport: A career transitions perspective. In E. Tsung-Min Hung, R. Lidor, \& D. Hackfort (Eds.), Psychology of sport excellence (pp. 63-74). Morgantown, WV: Fitness Information Technology.

Stambulova, N., \& Alfermann, D. (2009). Putting culture into context: Cultural and cross-cultural perspectives in career development and transition research and practice. International Journal of Sport and Exercise Psychology, 7(3), 292-308.

Stambulova, N., Alfermann, D., Statler, T., \& Côté, J. (2009). Career development and transitions of athletes: The ISSP position stand. International Journal of Sport \& Exercise Psychology, 7, 395-412.

Tanggaard, L. (2006). A psychological field study of learning: Analysis of methodological aspects. Nordic Psychology, 58, 196-214. methodological aspects. Nordic Psychology, 58, 196
doi:http://dx.doi.org/10.1027/1901-2276.58.3.196

Yin, R. K. (2009). Case study research: Design and methods (4th ed). Thousand Oaks, CA: Sage Publications. 